isid/ms/2009/10

October 16, 2009

http://www.isid.ac.in/statmath/eprints

\title{
An asymmetric kernel estimator of density function for stationary associated sequences
}

\author{
Yogendra P. Chaubey \\ ISHA DEWAN
}

Indian Statistical Institute, Delhi Centre 7, SJSS Marg, New Delhi-110 016, India 



\title{
AN ASYMMETRIC KERNEL ESTIMATOR OF DENSITY FUNCTION FOR STATIONARY ASSOCIATED SEQUENCES
}

\author{
Yogendra P. Chaubey and Isha Dewan \\ Concordia University and Indian Statistical Institute
}

December 17, 2009

\begin{abstract}
Consider a sequence of stationary non-negative associated random variables with common marginal density $f(x)$. Here we use the empirical survival function as studied in Bagai and Prakasa Rao (1991) and apply the smoothing technique proposed by Chaubey et al. (2007). The resulting smooth estimators of the density function $f$ and that of the corresponding distribution function are shown to inherit the asymptotic properties similar to those obtained in Bagai and Prakasa Rao (1995) for the kernel-type density estimators.
\end{abstract}

Key words and phrases: Associated sequence, asymmetric kernel estimator, strong consistency, survival function

AMS 1980 subject classification: 62G07, 62G20

\section{Introduction}

Let $\left\{X_{n}, n \geq 1\right\}$ be a strictly stationary sequence of associated random variables defined on a probability space $(\Omega, \mathcal{F}, \mathcal{P})$. A set of random variables $\left\{X_{1}, \ldots, X_{n}\right\}$ is said to be associated (cf. Esary, Proschan and Walkup (1967)) if for every pair of functions $h(\mathbf{x})$ and $g(\mathbf{x})$ from $R^{n}$ to $R$, which are nondecreasing componentwise,

$$
\operatorname{Cov}(h(\mathbf{X}), \mathbf{g}(\mathbf{X})) \geq \mathbf{0}
$$


whenever it is finite, where $\mathbf{X}=\left(X_{1}, X_{2}, \ldots, X_{n}\right)$. An infinite sequence $\left\{X_{n}\right\}$ of random variables is said to be associated if every finite subset is associated.

Let $f, F$ and $\bar{F}$, respectively, denote the density function, the distribution function and the survival function of $X_{1}$. When the observations are i.i.d., several authors have suggested density estimators based on kernels, histogram methods, orthogonal functions, etc, (see, e.g. Prakasa Rao (1983), Devroye (1989) and Wand and Jones (1995)).

The most commonly used estimator of the density function is the kernel estimator (Rosenblatt(1956), Parzen (1962)) given by

$$
f_{n}(x)=\frac{1}{n h_{n}} \sum_{i=1}^{n} k\left(\left(x-X_{i}\right) / h_{n}\right),
$$

where $k$ is the kernel function, which is generally a symmetric density function with mean zero and variance 1 and $h_{n}$ is the bandwidth. Silverman (1986) noticed that in case of positive valued random variables this estimator assigns positive mass for $x$ in the interval $(-\infty, 0)$. Besides at times there is difficulty in estimating discontinuity at the boundary. In order to avoid the first problem, Bagai and Prakasa Rao (1995) suggested the use of a kernel $k$ which is defined only on the positive part of the real line. However, this approach makes use of only the first $r$ order statistics for the value of $x$ in $\left[X_{r: n}, X_{(r+1): n}\right)$ where $X_{i: n}$ denotes the $i^{\text {th }}$ order statistic from the random sample $\left\{X_{1}, \ldots, X_{n}\right\}$. Chaubey and Sen (1996) suggested a density estimator based on Hille's smoothing lemma (see Feller (1965), pp. 229) which is the derivative of a smooth version of the empirical distribution function that uses the whole data. This has been extended to the case of associated data (see Chaubey and Dewan (2009)). However, it may not be quite appropriate at the lower most boundary for removing the bias. Chaubey, Sen and Sen (2007) proposed an estimator based on a generalization of Hille's lemma along with a perturbation idea, that is appropriate to take care of the boundary bias. This type of estimator was proposed earlier in Gawronski and Stadmüller (1981) [see also Stadmüller (1983)] for the iid case, but the perturbation idea to take care of boundary bias and the representation given in (3.2) seems new.

Bagai and Prakasa Rao (1995) studied the kernel type density estimators for associated sequences and showed that the kernel type estimator is strongly consistent, pointwise as well as uniformly, over certain sets. Roussas (1991) studied strong uniform consistency of kernel estimates of $r-t h$ order derivative of $f$ under some regularity conditions on the kernel and the bandwidth.

Here we study the estimator proposed by Chaubey et al. (2007) when the underlying 
random variables are stationary associated. Some prelimnary results are stated in section 2. In section 3 we propose a smooth estimator of the distribution function and study its strong consistency and asymptotic normality. Properties of a smooth estimator of the density function when underlying random variables are nonnegative are studied in section 4 .

\section{Preliminaries}

Here we put together results for associated random variables which are useful to study the properties of the proposed density estimators.

Bagai and Prakasa Rao (1991) studied the strong consistency, pointwise and uniform, of the empirical distribution function based on stationary associated random variables. They proved the following result.

Theorem 2.1 Let $\left\{X_{n}, n \geq 1\right\}$ be a stationary sequence of associated random variables with bounded continuous density for $X_{1}$. Assume that, for some $r>1$,

$$
\sum_{j=n+1}^{\infty}\left\{\operatorname{Cov}\left(X_{1}, X_{j}\right)\right\}^{1 / 3}=O\left(n^{-(r-1)}\right) .
$$

Then, for any compact subset $J \in R$,

$$
\sup \left[\left|F_{n}(x)-F(x)\right|: x \in J\right] \rightarrow 0 \text { a.s. as } n \rightarrow \infty \text {. }
$$

We will also need the following theorems in the proofs.

Theorem 2.2 (Bullinski (1996)) Let $X_{1}, X_{2}, \ldots, X_{n}$ be associated random variables - Let $h(x)$ be a continuous function from $R^{n}$ to $R$ such that for any $x \in R^{n}$ and any $k=1, \ldots, n$, there exist finite derivatives $\left(\partial^{+} h(x) / \partial x_{k}\right)$ and $\left(\partial^{-} h(x) / \partial x_{k}\right)$. Further suppose hat for each $k=1, \ldots, n$, there are at most a finite number of points at which $\left(\partial^{+} h(x) / \partial x_{k}\right) \neq\left(\partial^{-} h(x) / \partial x_{k}\right)$. Let

$$
L_{k}(h)=\max \left\{\left\|\frac{\partial^{+} h(x)}{\partial x_{k}}\right\|_{\infty},\left\|\frac{\partial^{-} h(x)}{\partial x_{k}}\right\|_{\infty}\right\}
$$


where $\|$.$\| stands for L^{\infty}$ norm. Further suppose that similar assumptions hold for another function $g$. Then, for any two disjoint subsets, $I$ and $J$ of $\{1, \ldots, n\}$, we have

$$
\mid \operatorname{Cov}\left(h\left(X_{i}, i \in I\right), g\left(X_{j}, j \in J\right) \mid \leq \sum_{i \in I} \sum_{j \in J} L_{i}(h) L_{j}(g) \operatorname{Cov}\left(X_{i}, X_{j}\right)\right.
$$

Theorem 2.3 (Newman (1980)) Let $\left\{X_{n}, n \geq 1\right\}$ be a stationary sequence of associated random variables with $E\left[X_{1}^{2}\right]<\infty$ and $0<\sigma^{2}=\operatorname{Var}\left(X_{1}\right)+2 \sum_{j=2}^{\infty} \operatorname{Cov}\left(X_{1}, X_{j}\right)<$ $\infty$. Then

$$
n^{-1 / 2} \frac{\sum_{j=1}^{n}\left(X_{j}-E\left(X_{j}\right)\right)}{\sigma} \stackrel{\mathcal{L}}{\rightarrow} N(0,1) \text { as } n \rightarrow \infty .
$$

Theorem 2.4 (Bagai and Prakasa Rao (1995)) For every $\alpha \in J$, an index set, let $\left\{X_{j}(\alpha), j \geq 1\right\}$ be an associated sequence. Let $f_{n}, n \geq 1$ be functions of bounded variation which are differentiable and suppose that $\sup _{n \geq 1} \sup _{x}\left|f_{n}^{\prime}(x)\right| \leq c<\infty$. Let $E\left(f_{n}\left(X_{j}(\alpha)\right)\right)=0$ for every $n \geq 1, j \geq 1$ and $\alpha \in J$. Suppose there exist $r>2$ and $\delta>0$ (independent of $\alpha, j, n$ ) such that

$$
\sup _{n \geq 1} \sup _{\alpha \in J} \sup _{j \geq 1} E\left|f_{n}\left(X_{j}(\alpha)\right)\right|^{r+\delta} \leq \infty .
$$

Let

$$
u(n, \alpha)=\sup _{k \geq 1} \sum_{j:|j-k| \geq n} \operatorname{Cov}\left(X_{j}(\alpha), X_{k}(\alpha)\right) .
$$

Suppose there exists $c>0$ independent of $\alpha \in J$ such that

$$
u(n, \alpha) \leq c n^{-(r-2)(r+\delta) / 2 \delta}
$$

Then, there exists a constant $B$ not depending on $n, m$ and $\alpha$, such that

$$
\sup _{m \geq 1} \sup _{\alpha \in J} \sup _{k \geq 0} E\left|S_{n+k, m}(\alpha)-S_{k, m}(\alpha)\right|^{r} \leq B n^{r / 2}
$$

where

$$
S_{m_{n}, \alpha}=\sum_{j=1}^{m_{n}} f_{n}\left(X_{j}(\alpha)\right)
$$




\section{A smooth estimator of the distribution function}

\subsection{General Support}

Chaubey et al. (2007) proposed the following smooth estimator of the distribution function, the motivation for which came from the generalization of Hille's Lemma (see Feller (1965), §6).

$$
\tilde{F}_{n}(x)=\int_{-\infty}^{\infty} F_{n}(t) d G_{x, n}(t)
$$

where $F_{n}(t)$ is the empirical distribution function based on $X_{1}, X_{2}, \ldots, X_{n}$, and $G_{x, n}, n=$ $1,2, \ldots$ is a family of distribution functions with mean $\mu_{n}(x) \rightarrow x$ and variance $v_{n}^{2}(x) \rightarrow 0$ as $n \rightarrow \infty$. Normally $G_{x, n}$ is chosen in such a way that it has the same support as that of the random variable under consideration. This will ensure that the estimator does not assign positive mass to undesired regions. One can write $\tilde{F}_{n}(x)$ as

$$
\tilde{F}_{n}(x)=1-\frac{1}{n} \sum_{i=1}^{n} G_{x, n}\left(X_{i}\right) .
$$

From the above equation, it is clear that $G_{x, n}(t)$ must be a decreasing function of $x$, so that $\tilde{F}_{n}(x)$ is a proper distribution function. It may be remarked that the above estimator reduces to the smooth estimator $F_{n K}(x)$ of $F(x)$ based on the kernel estimator in (1.1) given as

$$
F_{n K}(x)=\frac{1}{n} \sum_{i=1}^{n} K\left(\frac{x-X_{i}}{h_{n}}\right),
$$

when $G_{x, n}(t)=K\left((t-x) / h_{n}\right)$, and $K(x)=\int_{-\infty}^{x} k(u) d u$. This estimator has been investigated by Azzalini (1981) for the iid case. Its strong consistency in the case of associated random variables is proved in the following theorem.

Theorem 3.1 Let $\left\{X_{n}, n \geq 1\right\}$ be a stationary sequence of associated random variables with bounded continuous density for $X_{1}$. Assume that, for some $r>1$,

$$
\sum_{j=n+1}^{\infty}\left\{\operatorname{Cov}\left(X_{1}, X_{j}\right)\right\}^{1 / 3}=O\left(n^{-(r-1)}\right) .
$$

Then, for any compact subset $J \in R$,

$$
\sup \left[\left|\tilde{F}_{n}(x)-F(x)\right|: x \in J\right] \rightarrow 0 \text { a.s. as } n \rightarrow \infty .
$$


Proof : Notice that

$$
\left|\tilde{F}_{n}(x)-F(x)\right| \leq\left|\tilde{F}_{n}(x)-F_{n}^{*}(x)\right|+\left|F_{n}^{*}(x)-F(x)\right|,
$$

where

$$
F_{n}^{*}(x)=\int_{-\infty}^{\infty} F(t) d G_{x, n}(t) \rightarrow F(x) \text { as } n \rightarrow \infty .
$$

Further, for every $x$,

$$
\left|\tilde{F}_{n}(x)-F_{n}^{*}(x)\right| \leq \max _{t}\left|F_{n}(t)-F(t)\right| \int_{-\infty}^{\infty} d G_{x, n}(t)
$$

The result now follows using Theorem 2.1.

\subsection{Non-negative Support}

Next, we consider the following estimator proposed by Chaubey et al. (2007) when the support is $[0, \infty)$ where the the family $G_{x, n}$ is obtained from distribution function $Q_{v_{n}}(x)$ on $[0, \infty)$ that has mean 1 and variance $v_{n}^{2}$, namely,

$$
F_{n}^{+}(x)=1-\frac{1}{n} \sum_{i=1}^{n} Q_{v_{n}}\left(\frac{X_{i}}{x}\right)
$$

where $\nu_{n} \rightarrow 0$ as $n \rightarrow \infty$. Here $G_{x, n}(t)=Q_{v_{n}}\left(\frac{t}{x}\right)$.

Theorem 3.2 Let $\left\{X_{n}, n \geq 1\right\}$ be a stationary sequence of associated random variables with bounded continuous density for $X_{1}$. Assume that, for some $r>1$,

$$
\sum_{j=n+1}^{\infty}\left\{\operatorname{Cov}\left(X_{1}, X_{j}\right)\right\}^{1 / 3}=O\left(n^{-(r-1)}\right) .
$$

Let

$$
\begin{aligned}
L(x, t)= & \max \left[\frac{d^{+} Q_{v}(t / x)}{d t}, \frac{d^{-} Q_{v}(t / x)}{d t}\right] \\
& \left.\sup _{x} \sup _{t} L(x, t)\right)<A,
\end{aligned}
$$

where $A$ is a positive constant. Then, there exists a constant $C>0$ such that, for every $\epsilon>0$,

$$
\sup _{x} P\left[\left|F_{n}^{+}(x)-F_{n}^{*}(x)\right|>\epsilon\right] \leq C \epsilon^{-2 r} n^{-r}, \text { for every } n \geq 1,
$$

where

$$
F_{n}^{*}(x)=\int_{0}^{\infty} F(t) d Q_{v_{n}}(t)
$$


Proof: Let $Y_{j}(x)=1-Q_{v_{n}}\left(\frac{X_{i}}{x}\right)$.

$$
\sup _{x} \sup _{j}\left|Y_{j}(x)-E Y_{j}(x)\right| \leq 2 .
$$

Since $Y_{1}(x), Y_{2}(x), \ldots, Y_{n}(x)$ are increasing functions of associated random variables, they are associated. Furthermore,

$$
\sum_{j=n+1}^{\infty} \operatorname{Cov}\left(Y_{1}(x), Y_{j}(x)\right) \leq L(x, t) \sum_{j=n+1}^{\infty} \operatorname{Cov}\left(X_{1}, X_{j}\right) \leq A \sum_{j=n+1}^{\infty} \operatorname{Cov}\left(X_{1}, X_{j}\right) .
$$

Also, the condition (3.7) implies that $\operatorname{Cov}\left(X_{1}, X_{n}\right) \rightarrow 0$ as $n \rightarrow \infty$. Thus $\sup _{n} \operatorname{Cov}\left(X_{1}, X_{n}\right)<$ $\infty$ and it follows that

$$
\sum_{j=n+1}^{\infty} \operatorname{Cov}\left(X_{1}, X_{j}\right) \leq \sup _{n}\left\{\operatorname{Cov}\left(X_{1}, X_{n}\right)\right\}^{2 / 3} \sum_{j=n+1}^{\infty}\left\{\operatorname{Cov}\left(X_{1}, X_{j}\right)\right\}^{1 / 3} .
$$

Then from Eqs. (3.7), (3.10) and Theorem 2.4, it follows that, for every $n \geq 1$,

$$
\sup _{x} E \mid \sum_{j=1}^{n}\left(Y_{j}(x)-\left.E\left(Y_{j}(x)\right)\right|^{2 r} \leq C n^{r},\right.
$$

where $C$ is independent of $n$ and $x$. Then using Markov Inequality, we get that for every $\epsilon>0$,

$$
\sup _{x} P\left[\left|F_{n}^{+}(x)-F_{n}^{*}(x)\right|>\epsilon\right] \leq C \epsilon^{-2 r} n^{-r} .
$$

Using Borel-Cantelli Lemma, from the above theorem we have for every $x<\infty$, that

$$
\left|F_{n}^{+}(x)-F_{n}^{*}(x)\right| \rightarrow 0 \text { a.s. as } n \rightarrow \infty \text {. }
$$

However, since $\left|F_{n}^{*}(x)-F(x)\right| \rightarrow 0$ as $n \rightarrow \infty$, we have strong pointwise consistency of $F_{n}^{+}(x)$.

Corollary 3.1 Under the conditions of above theorem, for every $x$,

$$
F_{n}^{+}(x) \rightarrow F(x) \text { a.s. as } n \rightarrow \infty .
$$


The following theorem shows that the proposed estimator is asymptotically normal.

Theorem 3.3 Let $\left\{X_{n}, n \geq 1\right\}$ be a stationary sequence of associated random variables with $\sum_{j=2}^{\infty} \operatorname{Cov}\left(X_{1}, X_{j}\right)<\infty$. Then,

$$
\sqrt{n}\left(F_{n}^{+}(x)-F(x)\right) \stackrel{\mathcal{L}}{\rightarrow} N\left(0, \sigma^{2}(F)\right), \quad \text { as } n \rightarrow \infty,
$$

where $\sigma_{F}^{2}=\operatorname{Var}\left(Q\left(\frac{X_{1}}{x}\right)\right)+2 \sum_{j=2}^{\infty} \operatorname{Cov}\left(\left(Q\left(\frac{X_{1}}{x}\right), Q\left(\frac{X_{j}}{x}\right)\right)\right.$.

Proof: Recollect that $Y_{1}(x), Y_{2}(x), \ldots, Y_{n}(x)$ are stationary associated random variables. Also $\sum_{j=2}^{\infty} \operatorname{Cov}\left(\left(Q\left(\frac{X_{1}}{x}\right), Q\left(\frac{X_{j}}{x}\right)\right)<\infty\right.$.

The result then follows from Theorem 2.3.

\section{A smooth estimator of density function for non- negative associated sequence}

The distribution function estimator given in (3.6) leads to the following estimator of the density function.

$$
\frac{d}{d x}\left(F_{n}^{+}(x)\right)=\frac{1}{n x^{2}} \sum_{i=1}^{n} X_{i} q_{v_{n}}\left(\frac{X_{i}}{x}\right)
$$

where $q_{v}($.$) denotes the density corresponding to the distribution function Q_{v}($.$) .$

This estimator, however, may not be defined at $x=0$, except in cases $\lim _{x \rightarrow 0} \frac{d}{d x}\left(F_{n}^{+}(x)\right)$ exists. Besides, this limit could be zero. Hence the following modified version of the estimator is proposed as in Chaubey et al. (2007),

$$
f_{n}^{+}(x)=\frac{1}{n\left(x+\epsilon_{n}\right)^{2}} \sum_{i=1}^{n} X_{i} q_{v_{n}}\left(\frac{X_{i}}{x+\epsilon_{n}}\right), x \geq 0,
$$

with $\epsilon_{n}$ decreasing to 0 at a sufficiently slow rate as $n \rightarrow \infty$. Chaubey et al. (2007) have used the Gamma $\left.\left(\alpha=1 / v^{2}, \beta=v^{2}\right)\right)$ distribution function for $Q_{v_{n}}($.$) .$

The formula given above helps in computing the density estimator $f_{n}^{+}(x)$. However, we use the following integral representation to study the properties of the estimators,

$$
f_{n}^{+}(x)=\int_{0}^{\infty} F_{n}(t) \frac{d}{d x}\left[g_{x+\epsilon_{n}}(t)\right] d t
$$

where $g_{x, n}(t)=\frac{d}{d t} G_{x, n}(t)$. Bias of $f_{n}^{+}(x)$ : Following Chaubey et al. (2007) 


$$
\begin{aligned}
\operatorname{Bias}\left[f_{n}^{+}(x)\right] \\
=E\left[f_{n}^{+}(x)\right]-f(x) \\
=\frac{1}{\left(x+\epsilon_{n}\right)^{2}} E\left[X_{i} q_{v_{n}}\left(\frac{X_{i}}{x+\epsilon_{n}}\right)\right]-f(x) \\
=x f^{\prime}(x) \int_{0}^{\infty} y(y-1) q_{v_{n}}(y) d y+\epsilon_{n} f^{\prime}(x) \int_{0}^{\infty} y^{2} q_{v_{n}}(y) d y \\
\quad+o\left(\int_{0}^{\infty}\left[x y(y-1) q_{v_{n}}(y)+\epsilon_{n} y^{2} q_{v_{n}}(y)\right] d y\right) .
\end{aligned}
$$

Using the fact that

$$
v_{n}^{2}=\operatorname{Var}_{Q_{v_{n}}}(X)=E_{Q_{v_{n}}}\left[X_{1}\left(X_{1}-1\right)\right]
$$

and $v_{n}^{2}, \epsilon_{n} \rightarrow 0$ as $n \rightarrow \infty$, we get

$$
\operatorname{Bias}\left[f_{n}^{+}(x)\right]=\left(x v_{n}^{2}+\epsilon_{n}\right) f^{\prime}(x)+o\left(v_{n}^{2}+\epsilon_{n}^{2}\right) .
$$

Variance of $f_{n}^{+}(x)$ : Assume the following conditions as in Chaubey et al. (2007),

(A1) $\int_{0}^{\infty}\left(q_{v_{n}}(t)\right)^{m} d t=0\left(v_{n}^{-(m-1)}\right)$ as $v_{n} \rightarrow 0$ for $1 \leq m \leq 3$,

(A2) $I_{2}(q)=\lim _{v_{n} \rightarrow 0} v_{n} \int_{0}^{\infty}\left(q_{v_{n}}(t)\right)^{2} d t$ exists,

(A3) with $q_{m, v_{n}}^{*}(t)=\frac{\left(q_{v_{n}}(t)\right)^{m}}{\int_{0}^{\infty}\left(q_{v_{n}}(u)\right)^{m} d u,} \quad 1 \leq m \leq 3$, and as $v_{n} \rightarrow 0$

(i) $\mu_{m, v_{n}}=\int_{0}^{\infty} t q_{m, v_{n}}^{*}(t) d t=1+O\left(v_{n}\right)$

(ii) $\sigma_{m, v_{n}}^{2}=\int_{0}^{\infty}\left(t-\mu_{m, v_{n}}\right)^{2} q_{m, v_{n}}^{*}(t) d t=O\left(v_{n}^{2}\right)$

(iii) $\sup _{0<v_{n}<\epsilon_{n}} \int_{0}^{\infty} t^{4+\delta} q_{m, v_{n}}^{*}(t) d t<\infty$ for some $\delta>0, \epsilon>0$.

(A4) Let $\psi_{n}(x, y)=y q_{v_{n}}\left(\frac{y}{x+\epsilon_{n}}\right)$

$\frac{\partial}{\partial y} \psi_{n}(x, y)=q_{v_{n}}\left(\frac{y}{x+\epsilon_{n}}\right)+\frac{y}{x+\epsilon_{n}} q_{v_{n}}^{\prime}\left(\frac{y}{x+\epsilon_{n}}\right)$

Suppose $\sup _{x, y}\left|\frac{\partial}{\partial y} \psi_{n}(x, y)\right| \leq C$, where $C$ is a positive constant.

(A5) For all $\ell$ and $r \geq 0, \sum_{j:|\ell-j| \geq r} \operatorname{Cov}\left(X_{j}, X_{\ell}\right) \leq u(\ell)$ where $u(r)=e^{-\alpha r}$ for some $\alpha>0$ 
We now have

$$
\begin{aligned}
& \operatorname{Var}\left(f_{n}^{+}(x)\right)=\frac{1}{n\left(x+\epsilon_{n}\right)^{4}} \operatorname{Var}\left(X_{1} q_{v_{n}}\left(\frac{X_{1}}{x+\epsilon_{n}}\right)\right) \\
& +\frac{1}{n^{2}\left(x+\epsilon_{n}\right)^{4}} \sum_{1 \leq i \leq j \leq n} \sum_{j} \operatorname{Cov}\left(X_{i} q_{v_{n}}\left(\frac{X_{i}}{x+\epsilon}\right), X_{j} q_{v_{n}}\left(\frac{X_{j}}{x+\epsilon_{n}}\right)\right)
\end{aligned}
$$

Using Taylor's expansion of $q_{v_{n}}$ and assumptions (A1) to to (A3) it follows from Chaubey et al. (2007),

$$
\begin{aligned}
& \frac{1}{n} \operatorname{Var}\left(\frac{X_{1}}{\left(x+\epsilon_{n}\right)^{2}} q_{v_{n}}\left(\frac{X_{1}}{x+\epsilon_{n}}\right)\right) \\
& \quad \simeq \frac{I_{2}(q) f(x)}{n v_{n}\left(x+\epsilon_{n}\right)}+o\left(\left(n v_{n}\right)^{-1}\right) \text { as } v_{n} \rightarrow 0, \epsilon_{n} \rightarrow 0, n v_{n} \rightarrow \infty
\end{aligned}
$$

and

$$
\begin{aligned}
& \frac{2}{n^{2}\left(x+\epsilon_{n}\right)^{4}} \sum_{1 \leq i \leq j \leq n} \sum_{j \leq} \operatorname{Cov}\left(X_{i} q_{v_{n}}\left(\frac{X_{i}}{x+\epsilon_{n}}\right), X_{j} q_{v_{n}}\left(\frac{X_{j}}{x+\epsilon_{n}}\right)\right) \\
& \leq \frac{2 C}{n^{2}\left(x+\epsilon_{n}\right)^{4}} \sum_{1 \leq i \leq j \leq n} \sum_{1} \operatorname{Cov}\left(X_{i}, X_{j}\right) \quad(\text { by }(\mathrm{A} 4)) \\
& \leq \frac{C}{n\left(x+\epsilon_{n}\right)^{4}} . \quad(\text { By (A5) and stationarity ) }
\end{aligned}
$$

From (4.5), (4.6) and (4.7), we therefore get

$$
\begin{aligned}
\operatorname{Var} f_{n}^{+}(x)= & \frac{I_{2}(q) f(x)}{n v_{n}\left(x+\epsilon_{n}\right)}+O\left(\frac{1}{n\left(x+\epsilon_{n}\right)^{4}}\right) \\
& +o\left(\left(n v_{n}\right)^{-1}\right) \text { as } v_{n} \rightarrow 0, \epsilon_{n} \rightarrow 0, n v_{n} \rightarrow \infty .
\end{aligned}
$$

Mean Integrated Squared Error of $f_{n}^{+}(x)$ : Using above results

$$
\begin{aligned}
& M S E\left(f_{n}^{+}(x)\right)=\operatorname{Var}\left(f_{n}^{+}(x)\right)+\operatorname{Bias}^{2}\left[f_{n}^{+}(x)\right] \\
& =\frac{I_{2}(q) f(x)}{n v_{n}\left(x+\epsilon_{n}\right)}+\left[\left(x v_{n}^{2}+\epsilon_{n}\right) f^{1}(x)\right]^{2} \\
& \quad+O\left(\frac{1}{n\left(x+\epsilon_{n}\right)^{4}}\right)+o\left(v_{n}^{2}+\epsilon_{n}\right)+O\left(\left(n v_{n}^{-1}\right)\right) .
\end{aligned}
$$

Thus $f_{n}^{+}(x)$ is asymptotically unbiased and weakly consistent for $f(x)$. 
It also therefore follows that the mean integrated squared error is

$$
\begin{aligned}
\operatorname{MISE} & \left(f_{n}^{+}(x)\right)=\int_{0}^{\infty} \operatorname{MSE}\left(f_{n}^{+}(x)\right) d x \\
\simeq & \frac{I_{2}(q)}{n v_{n}} \int_{0}^{\infty} \frac{f(x)}{\left(x+\epsilon_{n}\right)} d x+\int_{0}^{\infty}\left[\left(x v_{n}^{2}+\epsilon_{n}\right) f^{\prime}(x)\right]^{2} d x \\
& +\int_{0}^{\infty} \frac{1}{n\left(x+\epsilon_{n}\right)^{4}} d x+o\left(v_{n}^{2}+\epsilon_{n}\right)+o\left(\left(n v_{n}\right)^{-1}\right) .
\end{aligned}
$$

The leading team of integrated mean square error is the asymptotic MISE,

$$
A M I S E\left[f_{n}^{+}\right]=\frac{I_{2}(q)}{n v_{n}} \int_{0}^{\infty} \frac{f(x)}{\left(x+\epsilon_{n}\right)} d x+\int_{0}^{\infty}\left[\left(x v_{n}^{2}+\epsilon_{n}\right) f^{\prime}(x)\right]^{2} d x+\int_{0}^{\infty} \frac{1}{n\left(x+\epsilon_{n}\right)^{4}} d x
$$

In the next theorem, we show that the proposed density estimator is pointwise consistent.

Theorem 4.1 Let $\left\{X_{n}, n \geq 1\right\}$ be a stationary sequence of associated random variables with bounded continuous density for $X_{1}$. Assume that, for some $r>1$

$$
\begin{aligned}
& \sum_{j=n+1}^{\infty}\left\{\operatorname{Cov}\left(X_{1}, X_{j}\right)\right\}^{1 / 3}=O\left(n^{-(r-1)}\right) \\
& v_{n} \rightarrow 0, \epsilon_{n} \rightarrow 0 \text { as } n \rightarrow \infty \\
& \sup _{x \geq 0} \int_{0}^{\infty} \mid \frac{\partial}{d x}\left[g_{x+\epsilon_{n, n}}(t)\right]=O\left(\left(\frac{\log \log n}{n^{1 / 2}}\right)\right) \\
& \sup _{u>0, v_{n}>0} u q_{v_{n}}(u)<\infty \\
& f(\cdot) \text { is Lipschitz continous on }[0, \infty) .
\end{aligned}
$$

Then, for any compact set $J \subset R$,

$$
\sup \left[\left|f_{n}(x)-f(x)\right| x \in J \mid \rightarrow 0 \text { a.s. as } n \rightarrow \infty\right. \text {. }
$$

Proof: $\quad$ By Fubini's theorem

$$
\begin{aligned}
f_{n}^{+}(x) & =\frac{d}{d x} \int_{0}^{\infty} F_{n}(t) g_{x+\epsilon_{n, n}}(t) d t \\
& =\int_{0}^{\infty} F_{n}(t)\left[\frac{d}{d x} g_{x+\epsilon_{n, n}}(t)\right] d t
\end{aligned}
$$


Therefore,

$$
\begin{aligned}
f_{n}^{+}(x)-f(x) & =\int_{0}^{\infty}\left(F_{n}(t)-F(t)\right)\left[\frac{d}{d x} g_{x+\epsilon_{n, n}}(t)\right] d t \\
& +\int_{0}^{\infty} F(t)\left[\frac{d}{d x} g_{x+\epsilon_{n, n}}(t)\right] d t-f(x) .
\end{aligned}
$$

Since $G_{x, n}(t)=Q_{v_{n}}\left(\frac{t}{x}\right), g_{x, n}(t)=\frac{1}{x} q_{v_{n}}(t / x)$, by Fubini's theorem, we get

$$
\begin{aligned}
\int_{0}^{\infty} & F(t)\left(\frac{d}{d x} g_{x+\epsilon_{n, n}}(t)\right) d t \\
& =\int_{0}^{\infty} F(t) \frac{d}{d x}\left(\frac{d}{d t} Q_{v_{n}}\left(\frac{t}{x+\epsilon_{n}}\right)\right) d t \\
& =\int_{0}^{\infty}\left(\frac{d}{d x} Q_{v_{n}}\left(\frac{t}{x+\epsilon_{n}}\right)\right) d F(t) \\
& =\int_{0}^{\infty} \frac{t}{\left(x+\epsilon_{n}\right)^{2}} q_{v_{n}}\left(\frac{t}{x+\epsilon_{n}}\right) f(t) d t . \\
& =E\left(f_{n}^{+}(x)\right) .
\end{aligned}
$$

Hence

$\left|f_{n}^{+}(x)-f(x)\right| \leq \sup _{t}\left|F_{n}(t)-F(t)\right| \int_{0}^{\infty}\left|\frac{d}{d x} g_{x+\epsilon_{n}, n}(t)\right| d t+\int_{0}^{\infty}\left|f\left(t\left(x+\epsilon_{n}\right)\right)-f(x)\right| t q_{v_{n}}(t) d t$.

From B1 and B3, it follows that the first term on the right in the above inequality converges to zero almost surely. As in Chaubey et al. (2007) from (B2), (B4), (B5), it follows that the second term also converges to zero. Hence the result follows.

\section{References}

[1] Azzalini, A. (1981). A note on the estimation of a distribution function and quantiles. Biometrika 68, 326-328.

[2] Bagai, I. and Prakasa Rao, B.L.S. (1991). Estimation of the survival function for stationary associated processes. Statistics and Probability Letters 12, 385-391.

[3] Bagai, I. and Prakasa Rao, B.L.S. (1995). Kernel-type density and failure rate estimation for associated sequences. Ann. Inst. Satatist. Math. 47, 253-266. 
[4] Bagai, I. and Prakasa Rao, B.L.S. (1995). Kernel type density estimates for positive valued random variables. Sankhyā A57, 56-67.

[5] Bullinski, A. V. (1996). On the convergence rates in the CLT for positively and negatively dependent random fields. In Probability Theory and Mathematical Statistics, Eds.: Ibragimov, I. A. and Zatsev, A. Yu. Gordon and Breach, UK, 2-14.

[6] Chaubey, Y. P. and Dewan, I. (2009). Smooth estimation of survival and density functions for a stationary associated process using Poisson weights. Technical Report No. 3/09, Department of Mathematics and Statistics, Concordia University, Montreal, Canada.

[7] Chaubey, Y. P., Sen, A. and Sen, P. K. (2007). A new smooth density estimator for non-negative random variables. Technical Report No. 1/07, Department of Mathematics and Statistics, Concordia University, Montreal, Canada.

[8] Chaubey, Y.P. and Sen, P.K. (1996). On smooth estimation of survival and density functions. Statistics and Decisions 14, 1-22.

[9] Dewan, I. and Prakasa Rao, B.L.S. (1999). A general method of density estimation for associated random variables. Journal of Nonparametric Statistics 10, 405-420.

[10] Devroye, L. (1989). A Course in Density Estimation. Birkhäuser, Boston.

[11] Esary, J., Proscahn, F. and Walkup, D. (1967). Association of random variables with applications. Ann. Math. Statist. 38, 1466-1474.

[12] Feller, W. (1965). An Introduction to Probability Theory and its Applications, Vol. II. John Wiley \& Sons, New York.

[13] Gawronski, W. and Stadmüller, U. (1981). Smoothing of histograms by means of lattice- and continuous distributions. Metrika 28, 155-164.

[14] Newman, C. M. (1980). Normal fluctuations and the FKG inequalities. Comm. Math. Phys. 74, 119-128.

[15] Parzen, E.(1962). On estimation of probability density and mode. Ann. Math Statist. 33, 1065-1070. 
[16] Prakasa Rao, B. L. S. (1983). Nonparametric Functional Estimation, Academic Press, New York.

[17] Rosenblatt, M. (1956). Remarks on some nonparametric estimates of density functions. Ann. Math Statist. 27, 832-837.

[18] Roussas, G. G. (1999). Positive ans negative dependence with some statistical applications. In Nonparametrics and Time Series, Ed.: S. Ghosh, Marcel Dekker, New York, $757-788$.

[19] Silverman, B. W. (1986). Density Estimation for Statistics and Data Analysis, Chapman and Hall, London.

[20] Stadmüller, U. (1983) Asymptotic Distributions of Smoothed Histograms. Metrika 30, $145-158$.

[21] Wand, M.P. and Jones, M. C. (1995). Kernel Smoothing, Chapman \& Hall, New York. 